\title{
Research on Hardware In-the-Loop Simulation System of Nuclear Power Station Controller Board in Alpha-Stable Noise
}

\author{
Xincai Chang ${ }^{1,}$, Jishi Guan ${ }^{1,2, b}$, Liangcai $\mathrm{Qi}^{1, \mathrm{c}}$ \\ ${ }^{1}$ China Nuclear Power Technology Research Institute Beijing Division \\ FL. $11^{\text {th }}$, CEC Building, No.6 south zhong guan cun St., Haidian District, Beijing, P.R.China \\ ${ }^{2}$ Jilin University, Nanling Campus 5988 Renmin StreetChangchun, Jilin, P.R.China \\ achangxincai@cgnpc.com.cn, bguanjishi@cgnpc.com.cn, qlc-acai@163.com
}

\begin{abstract}
Keywords: Hardware in-the-loop simulation, Alpha-Stable Noise, LabVIEW
Abstract. Nuclear power station PI controller board is used for complex nonlinear and adaptive control loop. The board realizes the classic PI control, eliminates the interference of the input signal may be produced. In this paper, alpha-stable noise is introduced into the input end of the controller circuit, the hardware in-the-loop simulation platform built by NI LabVIEW study the effect of alpha-stable noise on the PI controller circuit. The results show that alpha-stable noise can lead to closed loop controller system oscillation, make the system unstable.
\end{abstract}

\section{Introduction}

In the field of communication technology, traditionally the background signal noise is often used additive Gaussian distribution model to describe. In fact, this assumption is not accurate. It has been found that in the atmospheric noise, sonar and submarine communications in the acoustic signals and noise are very similar to Gaussian white noise distribution characteristics, but has thicker tail than Gaussian white noise [1]. Rappaport and Stuck found that this spike pulse characteristics, and has thick tail noise can be described by alpha-stable distribution [2]. Most of the existing development process using the actual equipment of various experiments. However improper input signal and control algorithm may cause irreversible damage to the controller board. Pure software simulation used for theoretical analysis and research, from the actual production, not much significant guidance.

The hardware-in-the-loop simulation (HILS) has been used in various kinds of engineering fields such as War industry, automobile manufacturing, robotics, and power electronics. HILS used to dev elop and analysis complex systems. HILS includes electrical simulations of actuators and Transmitter s. These electrical simulations are bridges between the software simulations and real objects [3]-[5]. I $\mathrm{n}$ order to solve the problem that the cost of hardware test is too high and the algorithm is not flexibl e enough, the HILS technology is applied to solve the problem [6].

In the NI LabVIEW environment, the alpha-stable noise signal is simulated by computer simulati on [7]. The input of the nuclear power station controller board is controlled by the NI 6259 card. An $\mathrm{d}$ the output AO signal of the board is collected and analyzed by LabVIEW. LabVIEW provides a co nvenient man-machine interface, which is convenient for the user to choose the control mode, set the control parameters, and real time acquisition and output waveforms [8].

\section{HILS system}

Nuclear power station controller board is a proportional plus integral controller. The connection between the board and the other components of the 9020 system of the nuclear power station can be used in the nonlinear and adaptive control circuit. If there is alpha-stable noise in the nuclear power plant control circuit, it will have a negative impact on nuclear power plant equipment. To test the effect of alpha-stable noise in the control loop of a nuclear power plant, the alpha noise is introduced into the control loop, which may damage the equipment. To this end, the hardware in the loop simulation platform is built, as shown in Fig. 1 


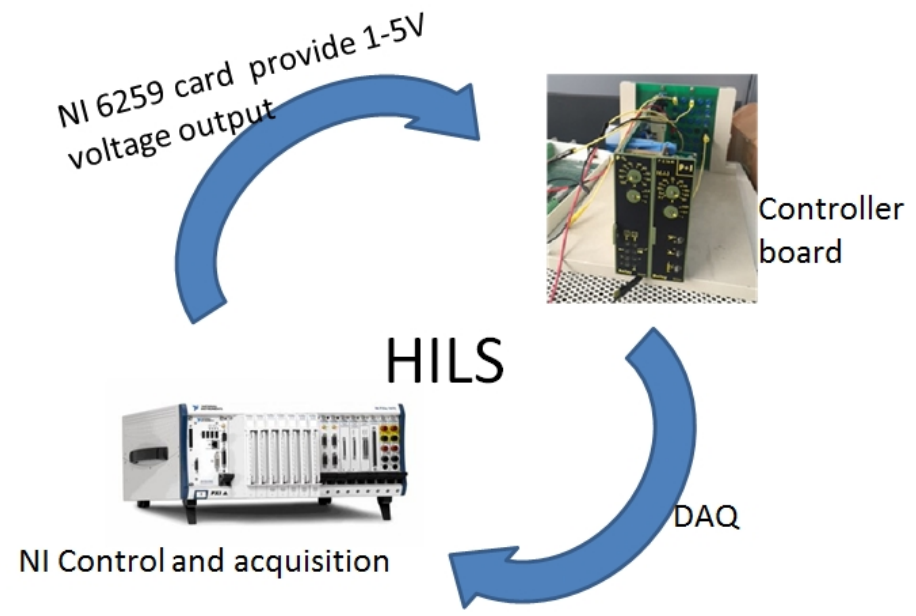

Fig. 1 Conceptual structure of HILS system

Fig. 2 shows a typical single loop control system, G (c) for the controller, $G(P)$ for the controlled object, $G(d)$ for the disturbance channel transfer function, $d$ for the disturbance, $y$ for the system output

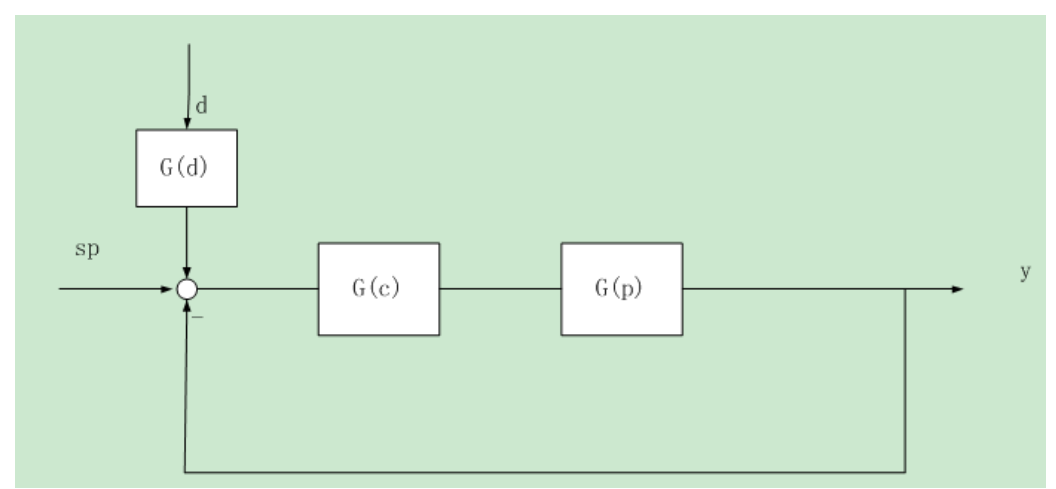

Fig. 2 Single loop control system

\section{LabVIEW Simulation}

The alpha noise is simulated by LabVIEW. Fig. 3-6 are the alpha-stable distributions of different alpha values of random variables in the sample sequence, are used $a=0, \beta=0, \gamma=1$ Standard $S \alpha S$ distribution. Sample number $\mathrm{N}=6000$.Fig. 7 is Gauss distribution of the sample sequence.

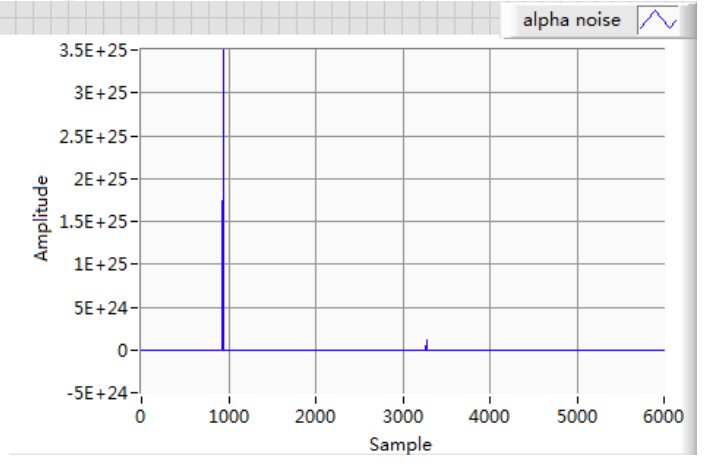

Fig. 3 Alpha $=0.3$

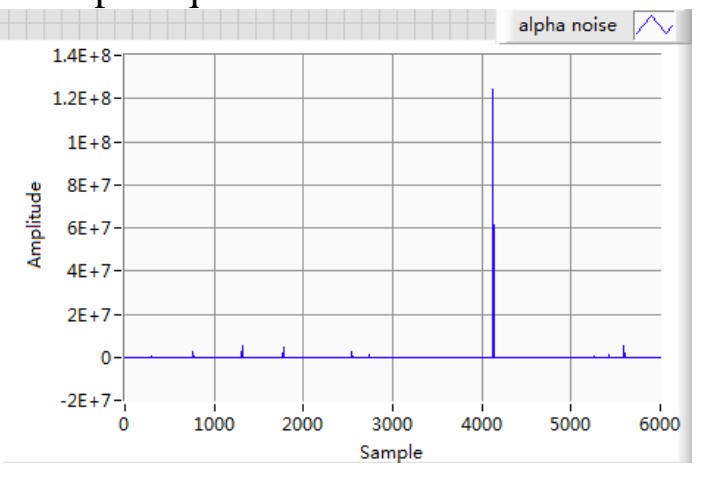

Fig. 4 Alpha $=0.6$ 


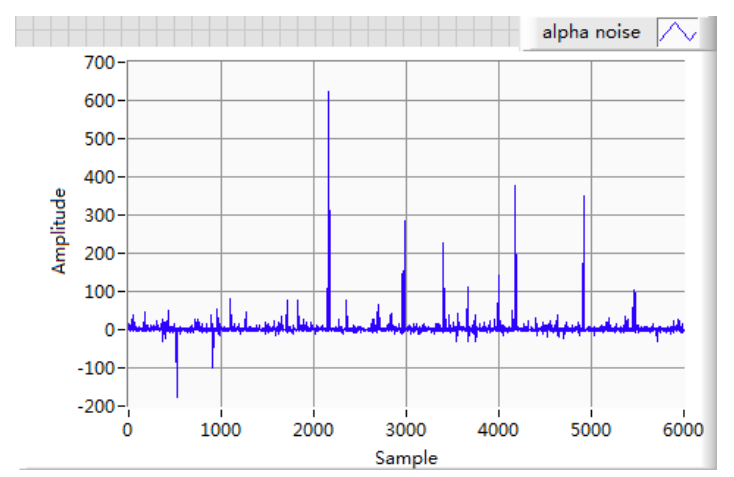

Fig. 5 Alpha=1.2

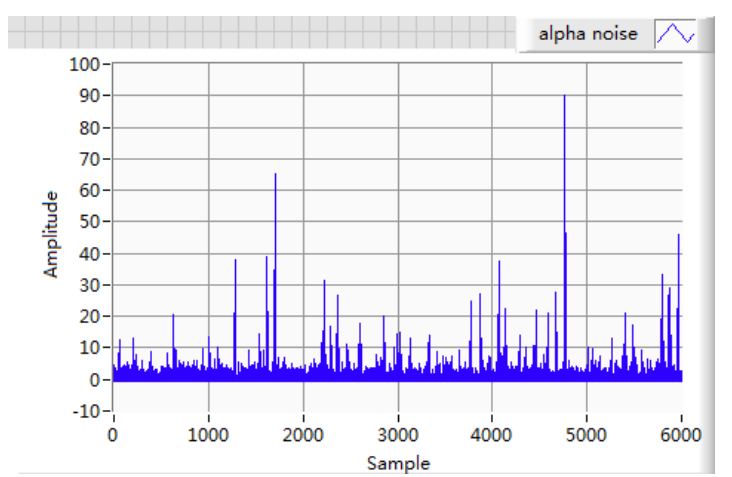

Fig. 6 Alpha=1.8

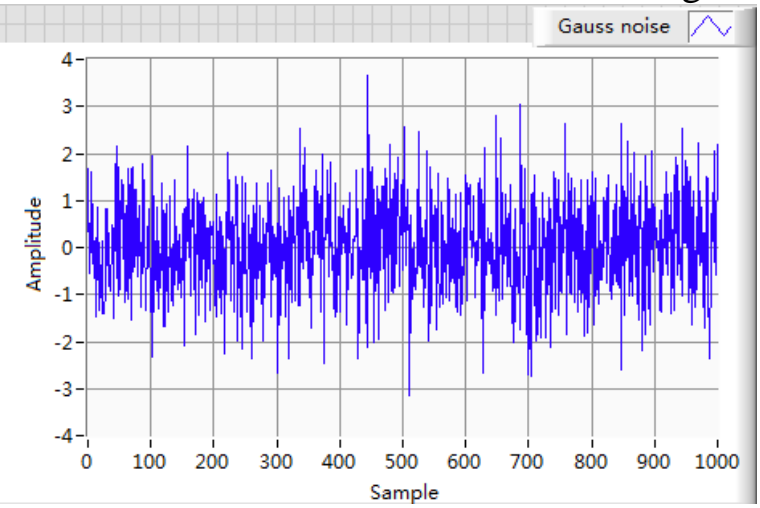

Fig. 7 Gauss distribution

It can be seen from the Fig. 3-6 that the larger the alpha value, the weaker the impulse characteristic of the signal. Fig. 7 shows that there is no obvious pulse characteristics of the Gauss distribution.

\section{Test Results}

The controller board of nuclear power station is acting on the first-order inertia link. The structure diagram is shown as Fig. 2. The time domain waveform of the output of the inertial link is collected by the NI data acquisition card 6259, as shown in Fig. 8.

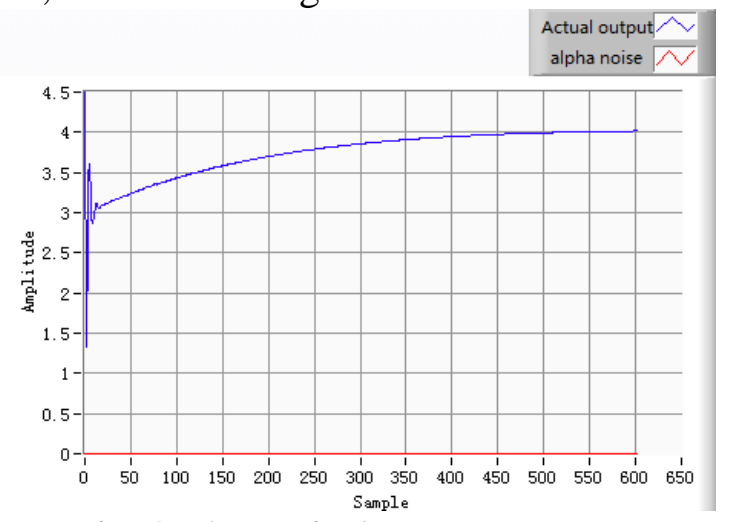

Fig. 8 Theoretical response curve

The Gaussian white noise and alpha signals are added to the input signal, the NI data acquisition card 6259 collects inertial response curve, as shown in Fig. 9-12 


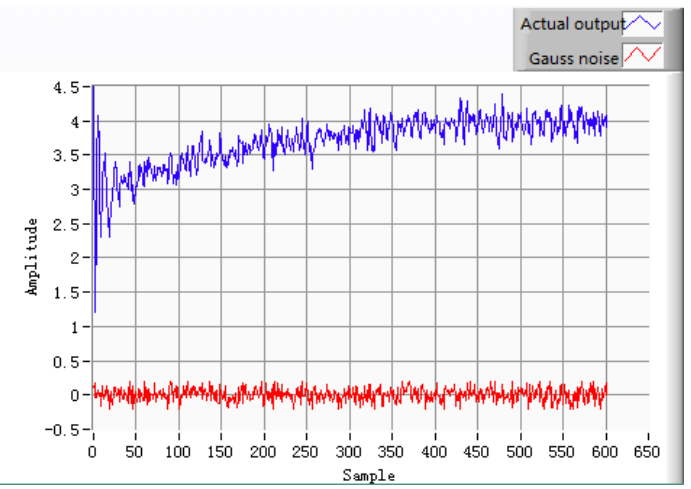

Fig. 9 Gauss noise response curve

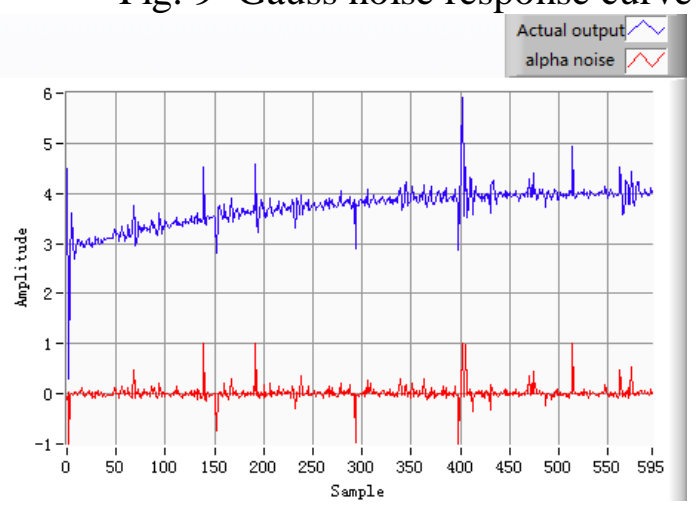

Fig. 11 Alpha=1.2 response curve

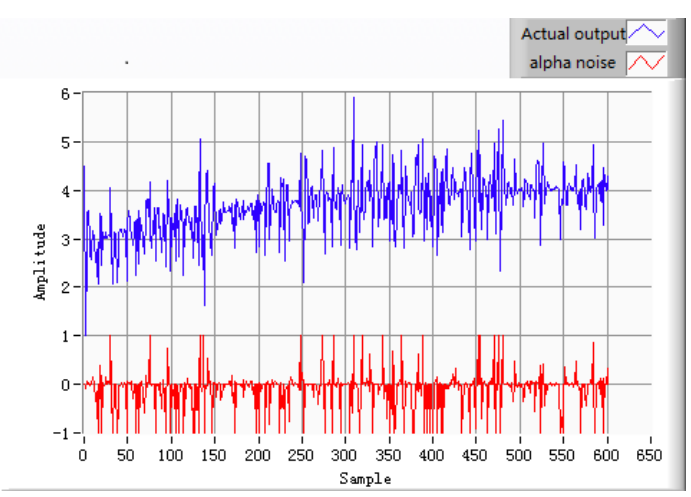

Fig. 10 Alpha=0.6 response curve

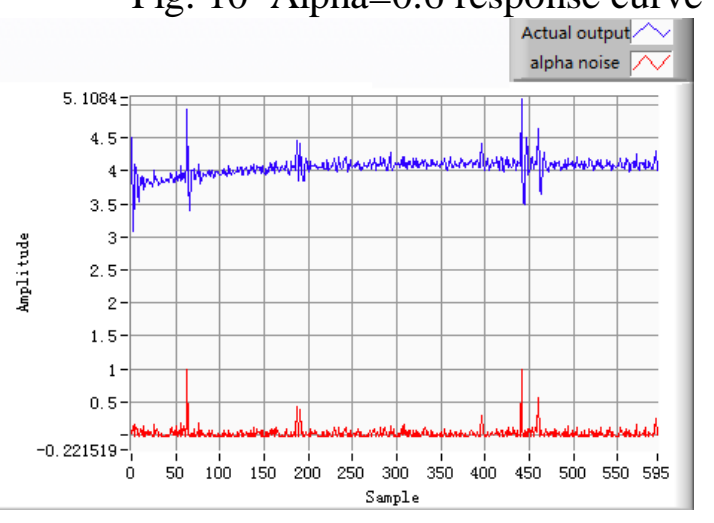

Fig. 12 Alpha=1.8 response curve

The effects of different alpha values on the system are different. The smaller the alpha value is, the greater the impact.

\section{Conclusions}

This paper proposed the hardware in-the-loop simulation system, which was used in the nuclear power station control loop. LabVIEW software simulated alpha-stable noise and Gauss noise, and acted them on the control loop. Nuclear power station controller board's inputs voltages were controlled by the LabVIEW software programming, which also controlled the input noise amplitudes, then the PI control card acted on the one order inertial link, finally NI 6259 card collected the response curve of the control loop. This verified the feasibility of hardware in-the-loop simulation system. The results showed that the alpha noise could lead to a closed loop controller system oscillation, making the system unstable. And the smaller the alpha value was, the greater the impact.

\section{References}

[1] Navia-Vazquez, A.;Arenas-Garcia, J.; submitted to IEEE Transactions on Signal Processing2012,60(3),1478-1482,5

[2] Marco J. Lombardi; Simon J. Godsill in IEEE Transactions on Signal Processing2006,54(2),775-779,5

[3] B. Lu, X.Wu, H. Figueroa, and A.Monti, "A low-cost real-time hardwarein-the-loop testing approach of power electronics controls," IEEE Trans.Ind. Electron, vol. 54, no. 2, pp. 919-931, Apr. 2007.

[4] W. Lee, M. Yoon, and M. Sunwoo, "A cost- and time-effective hardwarein-the-loop simulation platform for automotive engine control systems,"J. Automobile Eng., vol. 217, no. 1, pp. 41-52, 2002. 
[5] J. Du, Y.Wang, C. Yang, and H.Wang, "Hardware-in-the-loop simulationapproach to testing controller of sequential turbocharging system," inProc. 2007 IEEE Int. Conf. Autom. Logistics, Aug. 2007, pp. 2426-2431.

[6] Jeon, J.-H.;Kim, J.-Y.;Kim, H.-M.;Kim, S.-K.;Cho, C.;Kim, J.-M.;Ahn, J.-B.;Nam, K.-Y. submitted to IEEE Transactions on Power Electronics2010,25(12),2919-2929,11

[7] D. Luna-Moreno; Y.M. Espinosa S;In Optik - International Journal for Light and Electron Optics2015,126(19),1923-1929,7

[8] Andrzej Odon;Zbigniew Krawiecki;In Measurement (2011) 